\title{
New Basis Set for the Evaluation of Specific Rotation in Flexible Biological Molecules in Solution
}

\author{
Baranowska-Aczkowska, Angelika; aczkowski, Krzysztof Z.; Henriksen, Christian; Fernandez, Berta
}

Published in:

Journal of Physical Chemistry A

Link to article, DOI:

10.1021/acs.jpca.8b03320

Publication date:

2018

Document Version

Peer reviewed version

Link back to DTU Orbit

Citation (APA):

Baranowska-Aczkowska, A., aczkowski, K. Z., Henriksen, C., \& Fernandez, B. (2018). New Basis Set for the Evaluation of Specific Rotation in Flexible Biological Molecules in Solution. Journal of Physical Chemistry A, 122(24), 5477-5483. https://doi.org/10.1021/acs.jpca.8b03320

\section{General rights}

Copyright and moral rights for the publications made accessible in the public portal are retained by the authors and/or other copyright owners and it is a condition of accessing publications that users recognise and abide by the legal requirements associated with these rights.

- Users may download and print one copy of any publication from the public portal for the purpose of private study or research.

- You may not further distribute the material or use it for any profit-making activity or commercial gain

- You may freely distribute the URL identifying the publication in the public portal 


\title{
New Basis Set for the Evaluation of Specific Rotation in Flexible Biological Molecules in Solution
}

\author{
Angelika Baranowska-Łączkowska, ${ }^{*}{ }^{\dagger}$ (1) Krzysztof Z. Łączkowski, ${ }^{\ddagger}$ Christian Henriksen, ${ }^{\text {II }}$ \\ and Berta Fernández ${ }^{\S}$ \\ ${ }^{\dagger}$ Institute of Physics, Kazimierz Wielki University, Plac Weyssenhoffa 11, PL-85072 Bydgoszcz, Poland \\ ${ }^{\ddagger}$ Department of Chemical Technology and Pharmaceuticals, Faculty of Pharmacy, Collegium Medicum Nicolaus Copernicus \\ University, 2 Jurasz St., PL-85089 Bydgoszcz, Poland

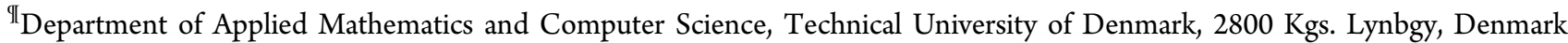 \\ ${ }^{\S}$ Department of Physical Chemistry, University of Santiago de Compostela, E-15782 Santiago de Compostela, Spain
}

ABSTRACT: A detailed theoretical investigation of specific rotation is carried out in solution for nine flexible molecules of biological importance. Systematic search for the main conformers is followed by time-dependent density functional theory (TD-DFT) calculations of specific rotation employing a wide range of basis sets. Due to conformational flexibility of the compounds under study, the possibility of basis set size reduction without deterioration of the results is investigated. The increasing size (d-)augcc-pVXZ (X = D, T, Q) bases of Dunning et al., and the ORP basis set, recently developed

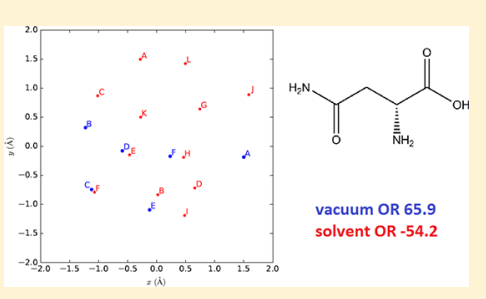
to efficiently provide molecular specific rotation, are used for this purpose. The polarizable continuum model is employed at all steps of the investigation. Comparison of the present results with the available data obtained in a vacuum reveals considerable differences, the values in solution being much closer to the experimental specific rotation data available. The ORP basis set proves to be competitive with the d-aug-cc-pVDZ set of Dunning in specific rotation calculations carried out in solution. While having the same number of functions, the former yields, in general, results considerably closer to the reference triple- $\zeta$ values. We can thus recommend the ORP basis set to study the optical rotation in conformationally flexible molecules in solution.

\section{INTRODUCTION}

The determination of the absolute configuration of chiral compounds is a crucial step in fields like asymmetric synthesis or drug design. Considering the difficulties experimental techniques, like X-ray crystallography or chemical correlation, have in the determination of the absolute configuration, the theoretical approach appears to be a very promising tool. Nevertheless, despite the fast progress in computing tools, accurate theoretical evaluation of optical rotation in flexible molecules still remains a challenge. In addition to the use of an appropriate electron correlation treatment, a thorough search of the conformational space is mandatory to start with. When the proper strategy is followed, data comparable to experimental results can be obtained in a much faster and efficient way. In order to carry out accurate optical rotation studies, the selection of an adequate correlation method and basis set is needed; to be able to compare the theoretical results to experimental values in solution, the evaluation of the property in solution is crucial.

Considering the correlation method, and since the majority of the chiral molecules that are usually found in living organisms are large in size, the use of the majority of the post-Hartree-Fock methods available in the most commonly used computational chemistry programs is prohibitive and using the time-dependent density functional theory (TD-DFT) becomes mandatory. Bearing in mind that shortcomings in TD-
DFT inevitably lead to inaccuracies in the results, employing basis sets that in an efficient way can cancel the correlation method defficiencies is essential, making the selection of the basis set a very important step in these studies. However, the above-mentioned large size of the molecules of interest prevents the comprehensive basis set studies of optical rotation, as the use of large and flexible bases, containing polarization and diffuse functions, soon becomes prohibitive. Thus, in practice, optical rotation calculations are usually performed with medium-size basis sets, with the correlation consistent aug-ccpVDZ basis set of Dunning and co-workers ${ }^{1-3}$ (the n-aug-ccpVXZ basis sets are denoted naVXZ throughout) being a commonly employed example. Nevertheless, recently it has been shown that the aVDZ set may be insufficient for accurate optical rotation evaluation and, in particular, that the values of methyloxirane and fluoro-oxirane specific rotation results obtained using the aVDZ set are substantially different from those obtained in larger basis sets. ${ }^{4,5}$

The theoretical calculation of specific rotation values needs the evaluation of the trace of the mixed electric dipolemagnetic dipole polarizability tensor. Thus, the use of electric property-oriented basis sets appears to be a natural solution to

Received: April 8, 2018

Revised: $\quad$ May 17, 2018

Published: May 24, 2018 
<smiles>C[C@H](N)Cc1ccccc1</smiles><smiles>C[C@@H]1Cc2ccc(O)c3c2[C@]2(CC=C[C@@H](O)[C@@H]2O3)CN1C</smiles><smiles>C[C@H]1OC(=O)C[C@H]1C(=O)O</smiles><smiles>COc1ccc2cc([C@@H](C)C(=O)O)ccc2c1</smiles><smiles>C[C@H](O)N=[N+]=[N-]</smiles>

7<smiles>CC[C@H](O)N=[N+]=[N-]</smiles>

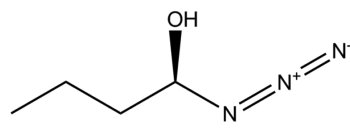

9

Figure 1. Molecules under investigation.

the problem of large basis set requirements and the resulting high cost accurate optical rotation calculations. Giorgio and coworkers have shown that the use of the Pol basis set can substantially improve $(S)$-methyloxirane specific rotation values with respect to the results obtained in the traditionally used aVDZ basis set. ${ }^{6}$ The Pol basis set employed in that work yielded values close to those obtained in the larger aVTZ and aVQZ bases. Additionally, the LPol-n basis sets can be recommended for optical rotation calculations, as they yielded specific rotation values of the model asymmetric methane molecule, methyloxirane, and fluoro-oxirane closer to the basis set limit than the larger size basis sets of Dunning and, thus, allowed reduction of the computational cost without deterioration of the results. ${ }^{5}$ Unfortunately, the B3LYP/LPol$\mathrm{n}$ results presented in ref 5 for $\beta$-pinene and trans-pinane were not confirmed in further studies. ${ }^{7-9}$

Despite the promising performance of the LPol-n sets in specific rotation investigations, a shortcoming was found; namely, numerical linear dependence of orbitals is encountered in the case of larger molecules, and as a result, a substantial number of basis functions is removed from the total basis set. ${ }^{8,9}$ The basis set size is thus decreased in a not fully controlled manner and may worsen the quality of the investigated system description. The problem of near-linear dependence of orbitals results from the method of generation of the LPol-n polarization functions, which have the same orbital exponent values as the p-type functions (s-type functions in the case of hydrogen) already present in the set. To reduce the problem of basis function near-linear dependence, we have recently developed a new compact polarized basis set for specific rotation calculations in large molecules within the TD-DFT approximation. ${ }^{9}$ This new set, referred to as ORP (acronym for optical rotation prediction), was generated for hydrogen, carbon, nitrogen, oxygen, and fluorine through addition of three uncontracted first-order polarization functions to the uncontracted VTZ basis set of Ahlrichs and co-workers ${ }^{10}$ and augmentation with one diffuse s-type and one diffuse p-type functions (one diffuse s-type function in the case of hydrogen) to additionally improve the description of a nucleus' distant regions. It has been employed in TD-DFT/B3LYP tests carried out in a vacuum for a set of conformationally rigid molecules. Specific rotation values obtained using the ORP basis set were in very good agreement with the reference data. Among Dunning's sets, the use of bases of at least the daVTZ level was necessary to obtain results of similar quality. The ORP results were very close to those obtained in the larger size LPol-n basis sets, while the ORP basis set is much more robust to near-linear dependence than the LPol-n sets. The ORP basis set was shown to be competitive also with respect to other small and medium-size basis sets.

Using TD-DFT and having as an aim the selection of a proper basis set to evaluate optical rotation in flexible biological molecules, recently we evaluated the specific rotation in nine molecules in a vacuum, namely, $(R)$-asparagine (molecule 1 ), $(S)$-amphetamine (molecule 2), (-)-morphine (molecule 3), $(2 S, 3 S)-(-)$-2-methyl-5-oxo-tetrahydro-3-furancarboxylic acid (molecule 4), methyl (2S,3S)-(-)-2-methyl-5-oxo-tetrahydro3-furancarboxylate (molecule 5), ( $S$ )-naproxen (molecule 6), (R)-1-azido-1-ethanol (molecule 7), (R)-1-azido-1-propanol (molecule 8), and (R)-1-azido-1-butanol (molecule 9). ${ }^{11}$ See Figure 1. For this we used the B3LYP functional and the ORP and Dunning augmented correlation consistent polarized valence basis sets.

We concluded that the ORP values were in good agreement with the aVTZ and aVDZ results and showed that the ORP basis set was a good choice to carry out optical rotation calculations in conformationally flexible molecules. These specific rotations in the above chiral azido alcohols were evaluated for the first time. In order to compare to the experimental values, solvent effects need to be taken into account, since they are expected to play a main role not only in the conformer analysis but also in the specific rotation of these flexible molecules. As an example, in ref 12 the optical rotation of molecules $\mathbf{4}$ and $\mathbf{5}$ has been evaluated both in a vacuum and in solution, and differences up to $24 \%$ and $36 \%$ were obtained between both sets of results, for 4 and 5 , respectively. Therefore, we decided to extend our previous study, where we only considered as reference vacuum values obtained at the 
Table 1. DFT(B3LYP)/6-31G** Relative Energies $\Delta E(\mathrm{kcal} / \mathrm{mol})$ and Conformer Populations $X(\%)^{a}$

\begin{tabular}{|c|c|c|c|c|c|c|c|c|}
\hline conformer & $\Delta E$ & $X$ & conformer & $\Delta E$ & $X$ & conformer & $\Delta E$ & $X$ \\
\hline & 1 & & & 4 & & $8 \mathrm{C}$ & 0.45 & 14.11 \\
\hline $1 \mathrm{~A}$ & 0.00 & 48.48 & $4 A$ & 0.00 & 42.97 & $8 D$ & 0.64 & 10.26 \\
\hline 1B & 0.56 & 18.80 & $4 B$ & 0.12 & 34.97 & $8 \mathrm{E}$ & 1.06 & 5.08 \\
\hline $1 \mathrm{C}$ & 0.92 & 10.19 & $4 \mathrm{C}$ & 0.67 & 13.86 & $8 F$ & 1.34 & 3.15 \\
\hline 1D & 1.31 & 5.31 & $4 D$ & 1.36 & 4.36 & $8 G$ & 1.38 & 2.94 \\
\hline $1 E$ & 1.37 & 4.81 & $4 E$ & 1.43 & 3.84 & $8 \mathrm{H}$ & 1.48 & 2.46 \\
\hline $1 F$ & 1.41 & 4.51 & & 5 & & $8 I$ & 1.63 & 1.94 \\
\hline $1 G$ & 1.76 & 2.48 & $5 A$ & 0.00 & 46.47 & $8 \mathrm{~J}$ & 1.73 & 1.61 \\
\hline $1 \mathrm{H}$ & 1.95 & 1.81 & $5 B$ & 0.25 & 30.71 & & 9 & \\
\hline $1 \mathrm{I}$ & 2.14 & 1.31 & $5 C$ & 0.73 & 13.63 & $9 A$ & 0.00 & 23.13 \\
\hline $1 \mathrm{~J}$ & 2.40 & 0.84 & $5 \mathrm{D}$ & 1.35 & 4.73 & $9 B$ & 0.07 & 20.62 \\
\hline $1 \mathrm{~K}$ & 2.41 & 0.83 & $5 E$ & 1.39 & 4.46 & $9 \mathrm{C}$ & 0.43 & 11.22 \\
\hline \multirow[t]{2}{*}{$1 \mathrm{~L}$} & 2.59 & 0.61 & & 6 & & 9D & 0.66 & 7.63 \\
\hline & 2 & & $6 \mathrm{~A}$ & 0.00 & 42.05 & $9 \mathrm{E}$ & 0.71 & 7.02 \\
\hline $2 A$ & 0.00 & 29.16 & $6 B$ & 0.22 & 29.14 & $9 F$ & 0.82 & 5.80 \\
\hline $2 B$ & 0.23 & 19.75 & $6 \mathrm{C}$ & 0.91 & 9.02 & 9G & 0.96 & 4.56 \\
\hline $2 \mathrm{C}$ & 0.42 & 14.25 & $6 \mathrm{D}$ & 1.18 & 5.70 & $9 \mathrm{H}$ & 1.18 & 3.14 \\
\hline $2 \mathrm{D}$ & 0.68 & 9.19 & $6 \mathrm{E}$ & 1.28 & 4.87 & 9I & 1.22 & 2.94 \\
\hline $2 \mathrm{E}$ & 0.75 & 8.25 & $6 \mathrm{~F}$ & 1.42 & 3.82 & $9 \mathrm{~J}$ & 1.31 & 2.53 \\
\hline $2 F$ & 0.83 & 7.16 & $6 \mathrm{G}$ & 1.52 & 3.21 & $9 \mathrm{~K}$ & 1.41 & 2.14 \\
\hline $2 \mathrm{G}$ & 0.98 & 5.61 & $6 \mathrm{H}$ & 2.19 & 1.04 & $9 \mathrm{~L}$ & 1.43 & 2.06 \\
\hline $2 \mathrm{H}$ & 1.00 & 5.41 & $6 \mathrm{I}$ & 2.51 & 0.61 & $9 M$ & 1.69 & 1.33 \\
\hline \multirow[t]{2}{*}{$2 I$} & 1.88 & 1.22 & $6 \mathrm{~J}$ & 2.57 & 0.55 & $9 \mathrm{~N}$ & 1.74 & 1.22 \\
\hline & 3 & & & 7 & & 90 & 1.78 & 1.16 \\
\hline $3 A$ & 0.00 & 62.74 & $7 \mathrm{~A}$ & 0.00 & 56.87 & $9 P$ & 1.92 & 0.90 \\
\hline $3 B$ & 0.38 & 32.82 & $7 B$ & 0.28 & 35.31 & $9 Q$ & 1.93 & 0.89 \\
\hline $3 C$ & 2.04 & 2.02 & $7 \mathrm{C}$ & 1.18 & 7.82 & 9R & 2.09 & 0.68 \\
\hline $3 D$ & 2.27 & 1.36 & & 8 & & $9 S$ & 2.25 & 0.51 \\
\hline \multirow[t]{2}{*}{$3 E$} & 2.42 & 1.06 & $8 \mathrm{~A}$ & 0.00 & 30.16 & $9 \mathrm{~T}$ & 2.26 & 0.51 \\
\hline & & & $8 B$ & 0.04 & 28.29 & & & \\
\hline
\end{tabular}

${ }^{a}$ See text for details.

daVTZ level, to the evaluation of the property in appropriate solvents, so that we are able to further improve our results and, when possible, compare them to experiment. Additionally, we investigate the possibility of basis set size reduction without deterioration of the specific rotation results. Such reduction is particularly important in the case of the large conformational flexibility of the compounds under study here, as the property computation has to be carried out for several stable conformers.

The manuscript is organized as follows: In section 2 we give the computational details, in section 3 we present and discuss the results, and in the last section we summarize and conclude.

\section{COMPUTATIONAL DETAILS}

The optical rotation $[\alpha]^{\lambda}$ of a conformationally flexible molecule $A$ at a given wavelength $\lambda$ is given by ${ }^{12}$

$$
[\alpha]^{\lambda}=\frac{e e\left(\epsilon_{\mathrm{A}}\right)}{100} \sum_{i=1}^{N} X_{i}[\alpha]_{i}^{\lambda}
$$

where $e e\left(\epsilon_{\mathrm{A}}\right)$ is the enantiomeric excess of enantiomer $\mathrm{A}$ in the mixture of enantiomers, $[\alpha]_{i}^{\lambda}$ is the optical rotation of the $i^{\text {th }}$ conformer, and $X_{i}$ is its fractional population defined as ${ }^{12}$

$$
X_{i}=\frac{\exp \left(-\Delta E_{i} / k T\right)}{\sum_{j=1}^{N} \exp \left(-\Delta E_{j} / k T\right)}
$$

Symbol $\Delta E_{i}$ denotes the relative energy of conformer $i, k$ is the Boltzmann constant, and $T$ is the temperature. Summations in eqs 1 and 2 run over all $N$ stable conformers of enantiomer $\mathrm{A}$.

The specific rotation is calculated at a wavelength $\lambda=589.3$ $\mathrm{nm}$ within the TD-DFT method using the B3LYP functional. This wavelength is selected because of being the most common at which OR is measured experimentally (sodium D-line), and the B3LYP functional after considering previous results in the literature. ${ }^{13-17}$ Calculations are carried out using Gauge invariant atomic orbitals (GIAOs) ${ }^{18}$ to get gauge-originindependent results. ${ }^{19,20}$

Solvent effects are considered both in the geometry optimizations and in the property calculations. For this we use the polarizable continuum model (PCM).$^{21-23}$ Depending on the system, the solvents are water (for molecule 1), methanol (for molecules 2-6), and trichloromethane (for molecules 7-9). Their dielectric constant values are equal to $78.3553,32.630$, and 4.7113 , respectively. In all cases, the cavity size radii are defined acording to the united atom topological model.

Reference theoretical results are obtained with the basis sets of Dunning and co-workers. Due to the large size and the conformational flexibility of the molecules under investigation, the use of very large basis sets is not possible, and therefore, we limit our study to the aVDZ, aVTZ, daVDZ, and the daVTZ bases; and in the case of molecules 3 and 6 to the aVDZ, and daVDZ sets. For molecules 1 and 7 aVQZ specific rotation is also evaluated. Additionally, for all systems, TD-DFT(B3LYP) 
properties are evaluated with the ORP basis set. In Table 2 the bases we used and the corresponding number of basis set functions are reported.

To obtain the conformers that contribute to the property, we followed the hybrid method described in detail in ref 11 , but here we additionally considered the solvent in the DFT conformer energy calculations. Moreover, on the basis of the results of tests carried out for molecules $\mathbf{4}$ and $\mathbf{5}$, and aiming at the best agreement with available experimental values, the 6$31 \mathrm{G}^{* *}$ basis set was chosen for optimization purposes. In this way, we get 12 stable conformers for system 1 , nine for system $\mathbf{2}$, five for 3, 4, and 5, ten for systems 6 and 8, three for system 7 , and 20 for system 9 . For all molecules vibrational frequency calculations are carried out to confirm that the optimized structures correspond to real minima on the potential energy surface.

All optical rotation results are given in $10^{-1} \mathrm{deg} \mathrm{cm}^{2} \mathrm{~g}^{-1}$ referred to as OR-units throughout.

Calculations are performed using the GAUSSIAN 09 package. $^{24}$

\section{RESULTS AND DISCUSSION}

The results are reported in Tables 1 and 2. Table 1 lists the main conformers obtained through DFT(B3LYP)/6-31G** level geometry optimizations. Both relative energies and conformer populations are reported. On the course of this study we reinvestigated vacuum conformers of molecule $\mathbf{1}$, finding an additional conformer with a population value equal to $3.8 \%$. This led to a proportional decrease in populations of

Table 2. PCM B3LYP Specific Rotation in OR Units ${ }^{a}$

\begin{tabular}{|c|c|c|c|c|c|c|}
\hline \multirow[b]{2}{*}{ molecule } & \multicolumn{6}{|c|}{ basis set } \\
\hline & aVDZ & aVTZ & aVQZ & daVDZ & daVTZ & ORP \\
\hline 1 & -65.3 & -58.0 & -54.2 & -69.2 & -57.9 & -42.2 \\
\hline vacuum & 60.9 & 65.6 & 65.9 & 62.5 & 66.5 & 76.5 \\
\hline$n$ & 279 & 598 & 1088 & 392 & 814 & 392 \\
\hline 2 & 22.8 & 26.0 & & 21.7 & & 27.3 \\
\hline vacuum $^{11}$ & 25.1 & 26.4 & & 24.4 & 27.4 & 28.3 \\
\hline$n$ & 347 & 759 & & 489 & 1036 & 489 \\
\hline 3 & -146.7 & & & -146.8 & & -143.9 \\
\hline vacuum $^{11}$ & -112.0 & & & -111.7 & & -109.5 \\
\hline$n$ & 654 & & & 919 & & 919 \\
\hline 4 & -113.0 & -113.5 & & -116.8 & -113.4 & -114.1 \\
\hline vacuum $^{11}$ & -82.2 & -82.4 & & -86.0 & -81.9 & -82.2 \\
\hline$n$ & 302 & 644 & & 424 & 876 & 424 \\
\hline 5 & -89.7 & -89.5 & & -94.7 & -89.9 & -90.8 \\
\hline vacuum $^{11}$ & -56.9 & -56.3 & & -61.4 & -56.7 & -56.6 \\
\hline$n$ & 343 & 736 & & 482 & 1002 & 482 \\
\hline 6 & 146.7 & & & 141.7 & & 151.7 \\
\hline vacuum $^{11}$ & 113.4 & & & 106.1 & & 112.3 \\
\hline$n$ & 517 & & & 726 & & 726 \\
\hline 7 & -125.2 & -139.4 & -138.9 & -123.4 & -139.9 & -141.6 \\
\hline vacuum $^{11}$ & -101.2 & -117.6 & -117.4 & -101.1 & -117.6 & -121.1 \\
\hline$n$ & 183 & 391 & 710 & 257 & 532 & 257 \\
\hline 8 & -187.6 & -193.2 & & -183.6 & -193.4 & -200.2 \\
\hline vacuum $^{11}$ & -144.5 & -152.7 & & -141.7 & -152.6 & -159.3 \\
\hline$n$ & 224 & 483 & & 315 & 658 & 315 \\
\hline 9 & -164.5 & -168.1 & & -160.6 & -168.2 & -178.0 \\
\hline vacuum $^{11}$ & -127.2 & -132.2 & & -124.5 & -132.4 & -140.8 \\
\hline$n$ & 265 & 575 & & 373 & 784 & 373 \\
\hline
\end{tabular}

${ }^{a}$ Vacuum values from ref 11 are also reported. $n$ is the number of basis set functions and $\lambda=589.3 \mathrm{~nm}$. See text for further details. the remaining five conformers with respect to the values reported in ref 11 . Total specfic rotation values evaluated in different basis sets including the new conformer are approximately $1.2-1.5$ OR units lower than those previously reported and are given in Table 2 .

We can observe considerable differences due to solutesolvent interactions, not only in the relative energy values but also in the conformers that have been found to be significant. For molecules 1, 3, 6, and 9 the number of conformers increases with respect to the vacuum case, in $6,2,2$, and 1 conformers, respectively, and only for molecules 7 and 8 is one less conformer located in solution.

For $\mathbf{1}$ in a vacuum the most stable conformer clearly dominated over the others with a population of 82.9 out of 100 ; now the situation is different, with two conformers having a considerably large population (48 and 19\%). A similar situation occurs in the case of molecule 3. For 2 in solution we have three conformers with populations larger than $10 \%$ (instead of 2 in a vacuum), and the same happens for molecule 4 . Populations obtained for 5, 6, and 8 are close to those we obtained in a vacuum. For molecules 7 and 9 the changes in conformer populations are larger; however, still the two lowest energy conformers are those that most contribute to the properties.

To get a more systematic insight into the influence of the solvent on the molecular geometries, we have carried out a systematic identification of similarities between the vacuum and the solvent structures. We define the distance between two conformer geometries $c_{1}, c_{2}$ as follows. Setting the mass of all atoms to 1 , we place $c_{1}$ and $c_{2}$ in the same coordinate system such that the center of mass is at the origin and such that the $x$ axis corresponds to the principal axis of the largest principal moment of inertia, the $y$-axis to the next principal axis, and the $z$-axis to the principal axis of the smallest principal moment of inertia. Then for each atom in $c_{1}$ we compute the distance to the closest atom in $c_{2}$, and vice versa for $c_{2}$, and we let $D\left(c_{1}, c_{2}\right)$ denote the largest of these distances. Since there are four ways to place $c_{2}$ such that the principal axes coincide with the $x$-, $y$-, and $z$-axis as described, we let $d\left(c_{1}, c_{2}\right)$ be the minimum of the four numbers $D\left(c_{1}, c_{2}\right)$. This gives us a metric on the set of conformers with distinct principal moments of inertia that is quick and convenient to compute. In this way, for each of the nine investigated molecules, the geometrical parameters of each conformer obtained in a vacuum were compared to those of all the conformers found in solution, and through an analysis of the distances between the atoms, the closest structure was obtained.

The largest distances (and therefore differences) between the atoms of the conformers in a vacuum and in solution are obtained for molecule $\mathbf{1}$, for which calculations have been carried out in water, the solvent with the highest polarity among those used in the present investigation, and therefore that with a largest influence on conformer structure. For DMSO, which is less polar than water and methanol, the distances between the atoms in the closest conformers in a vacuum and in solution are much shorter (on average, 0.14, 0.22 , and $0.25 \AA$ for molecules 7, 8, and 9, respectively). Taking into account the conformers with the largest populations in a vacuum and in DMSO, we can see a change in the conformer stabilities, but the conformer structures remain similar. This suggests that accounting for the presence of the solvent rather leads to some relative energy change for a given conformer than to large changes in the geometrical parameters. In particular, in 


\section{Molecule 1}

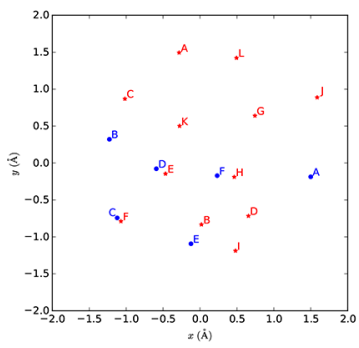

Molecule 4

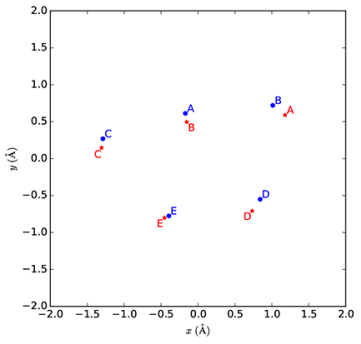

Molecule 7

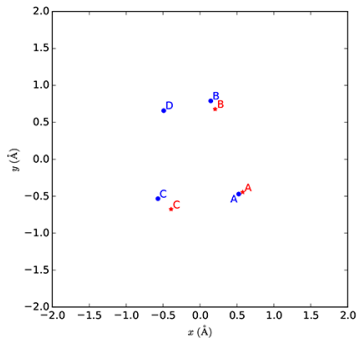

Molecule 2

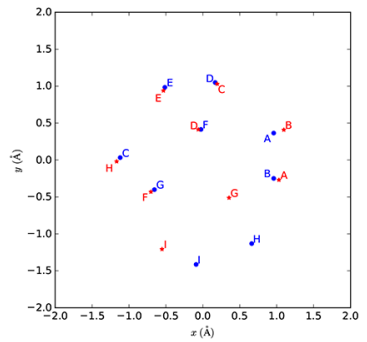

Molecule 5

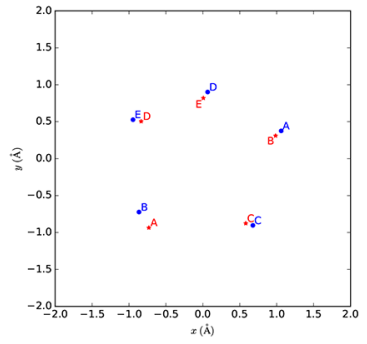

Molecule 8

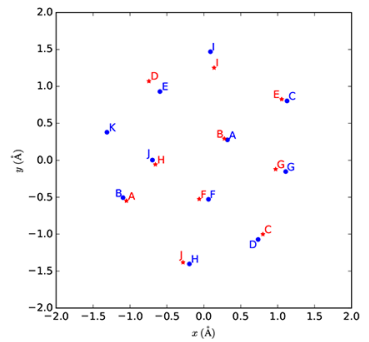

Molecule 3

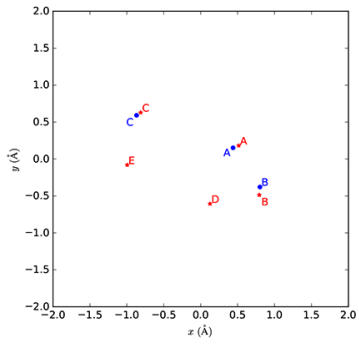

Molecule 6

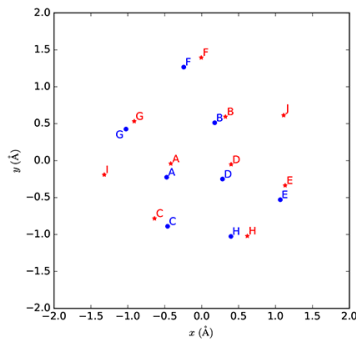

Molecule 9

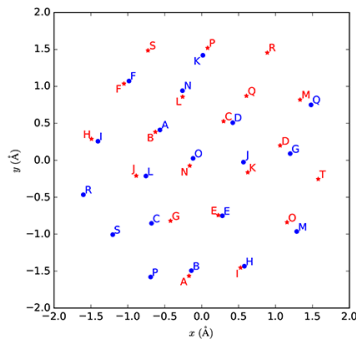

Figure 2. Visualization of distances between conformers. The blue points correspond to conformers in a vacuum whereas the red points correspond to conformers in solution.

molecule 7 the conformers remain almost the same as in a vacuum, and in $\mathbf{8}$ and $\mathbf{9}$ conformers 1 and 2 are interchanged in the energy rank, being the most stable conformer in DMSO the second most stable in a vacuum. Finally, in methanol, whose polarity is between those of the other two investigated solvents, the calculated distances between the closest atoms in the conformers in a vacuum and in solution are small (between 0.09 and $0.24 \AA$ on average). The stabilities of the two main conformers in solution either are interchanged with respect to those in a vacuum (molecules 2,4 , and 5 ) or have the same sequence (molecules 3 and 6). The influence of the solvent thus appears similar to that in the case of DMSO.

To visualize the found conformers, we think of each conformer as a point and look for an embedding $z_{i}=\left(x_{i}, y_{i}\right)$ of the points $c_{i}$ into the plane, so that the Euclidean distance $\| z_{i}$ - $z_{j}$ ll best resembles $d\left(c_{i}, c_{j}\right)$. This allows us to compare distances between conformers in a vacuum and a solvent at a glance. In practice, we employed a local minimum search to minimize

$$
\sum_{i<j}\left(\frac{\left\|z_{i}-z_{j}\right\|}{d\left(c_{i}, c_{j}\right)}-\frac{d\left(c_{i}, c_{j}\right)}{\left\|z_{i}-z_{j}\right\|}\right)^{2}
$$

using the positions obtained by classical multidimensional scaling as a starting guess. This yielded the embeddings illustrated in Figure 2.

In Table 2 we compare the B3LYP results we obtained for the specific rotation in solution, using the selected series of bases, to those available in a vacuum. ${ }^{11}$ In all the cases, but for molecule 2 , the results in solution are considerably different from those in a vacuum. For $\mathbf{2}$ we can see that at both the aVTZ and the ORP levels the specific rotation is about the same without and with the presence of the solvent. The ORP basis set (489 functions) gives slightly larger specific rotation than the aVTZ basis set (759 functions), but since this is also the case for the daVTZ basis set (1036 functions), we can conclude that the ORP basis set, with a considerable lower number of functions, seems to be more efficient than the aVTZ in the property evaluation.

For molecule 1 a large change, both in magnitude and in sign, is observed when the solvent is considered. It is the only system where a sign change in the property is obtained. Here the aVQZ (1088 functions) result is close to the daVTZ (814 functions), and the ORP basis set (392 functions) seems to underestimate the absolute value of the property.

Molecule 3 is the largest we studied, and therefore, we could only evaluate the specific rotation at the aVDZ, daVDZ, and ORP levels. The rotations are quite close in the three cases, negative and smaller with respect to the vacuum values. A 
similar situation occurs for system 6, but here the specific rotations are positive and larger than vacuum values. Additionally, in this case, we observe a larger difference between the aVDZ and the daVDZ results, being the former (517 functions) closer to the ORP (726 functions) than the latter (726 functions).

As far as we know, the only experimental data available are for molecules 4 and 5. Values of -106.7 and -82.8 OR units were reported in ref 12 . For both molecules the results are similar in all bases but the daVDZ, which seem to underestimate the rotation. This was also the case in a vacuum. Considering this, already at the aVDZ level the results look reasonable, and the number of basis functions is much smaller (302 and 343, for 4 and 5, respectively) than in the other cases. In comparison with the experimental values, the rotations are much closer to experiment when the solvent is considered in the calculations, in agreement with the earlier study in ref 12 and the theoretical results slightly underestimate the experimental values (by around $6 \%$ and $8 \%$, for 4 and 5 , respectively, at the daVTZ level).

For molecule 7 , as in the case of molecule 1, calculations in the aVQZ basis set (710 functions) were feasible, and therefore, we can take these results as reference. All Dunning's bases but the aVDZ and the daVDZ give similar results, and therefore, we can consider these rotations to be converged with respect to basis set improvement. The ORP basis set slightly overestimates the magnitude of the property, but it is much smaller than the triple and quadruple- $\zeta$ bases. Additionally, it gives considerably better results than the daVDZ, having the same number of functions.

Considering molecules 8 and $\mathbf{9}$, the daVTZ basis set was the largest employed in our calculations and these results are taken as reference. Bearing in mind that in all cases the differences between the daVTZ and the aVTZ results are negligible, showing that at the triple- $\zeta$ level and above double augmentation of the basis set is not necessary, we can also take the aVTZ values as reference. Noting that, in going to the aVQZ level (molecules 1 and 7), the specific rotation increases, we can conclude that the ORP basis set again slightly overestimates the magnitude of the rotation.

\section{SUMMARY AND CONCLUSIONS}

We study the specific rotation in the nine conformationally flexible molecules shown in Figure 1 in solution. For this, we use the TD-DFT method with the B3LYP functional, the aVXZ $(\mathrm{X}=\mathrm{D}, \mathrm{T}, \mathrm{Q})$ basis sets by Dunning et al., and the recently developed ORP basis set. The PCM model is employed to account for solvent effects.

Interaction with the solvent is shown to considerably change the magnitude ot the specific rotations. For the two cases where experimental specific rotations are available, much better agreement is obtained when the solvent is included in the calculations.

Considering the convergence of the results obtained in Dunning's basis sets, double augmentation at the triple- $\zeta$ level is not needed for the investigated systems. The ORP results agree reasonably well with the aVTZ and the aVQZ values, showing that the ORP basis set is a good choice for carrying out TD-DFT optical rotation calculations in conformationally flexible molecules, since it has the same size as the daVDZ basis set. Taking the results obtained in the (d)aVTZ or the aVQZ bases as reference, the ORP set competes with the larger bases in the case of molecules $2,4,5,7$, and 8 , although it does not for molecules $\mathbf{1}$ and 9. Yielding, in general, results closer to the basis set limit than the d-aug-cc-pVDZ set at no additional cost, the ORP set can be recommended to carry out optical rotation calculations for conformationally flexible molecules in solution.

\section{AUTHOR INFORMATION}

\section{Corresponding Author}

*A. Baranowska-Łaczkowska. E-mail: angelika.baranowska@ ukw.edu.pl.

\section{ORCID ${ }^{\circ}$}

Angelika Baranowska-Łączkowska: 0000-0001-9285-4991

\section{Notes}

The authors declare no competing financial interest.

\section{ACKNOWLEDGMENTS}

This work has been supported by Xunta de Galicia ("Axuda para Consolidación e Estruturación de unidades de investigación competitivas do Sistema Universitario de Galicia, ED431C 2017/17") and by the Foundation for Polish Science within the PARENT/BRIDGE programme (Pomost/2013-7/ 1), cofinanced from the European Regional Development Fund within the Innovative Economy Operational Programme. Centro de Supercomputación de Galicia (CESGA) is acknowledged for computational resources.

\section{REFERENCES}

(1) Dunning, T. H. Gaussian Basis Sets for Use in Correlated Molecular Calculations. I. The Atoms Boron through Neon and Hydrogen. J. Chem. Phys. 1989, 90, 1007-1023.

(2) Kendall, R. A.; Dunning, T. H.; Harrison, R. J. Electron Affinities of the First-Row Atoms Revisited. Systematic Basis Sets and Wave Functions. J. Chem. Phys. 1992, 96, 6796-6806.

(3) Woon, D. E.; Dunning, T. H. Gaussian Basis Sets for Use in Correlated Molecular Calculations. IV. Calculation of Static Electrical Response Properties. J. Chem. Phys. 1994, 100, 2975-2988.

(4) Pedersen, T. B.; Kongsted, J.; Crawford, T. D.; Ruud, K. On the Importance of Vibrational Contributions to Small-Angle Optical Rotation: Fluoro-Oxirane in Gas Phase and Solution. J. Chem. Phys. 2009, 130, 034310 .

(5) Baranowska, A.; Łączkowski, K. Z.; Sadlej, A. J. Model Studies of the Optical Rotation, and Theoretical Determination of its Sign for $\beta$ Pinene and trans-Pinane. J. Comput. Chem. 2010, 31, 1176-1181.

(6) Giorgio, E.; Rosini, C.; Viglione, R. G.; Zanasi, R. Calculation of the Gas Phase Specific Rotation of (S)-Propylene Oxide at $355 \mathrm{~nm}$. Chem. Phys. Lett. 2003, 376, 452-456.

(7) Mach, T. J.; Crawford, T. D. Basis Set Dependence of Coupled Cluster Optical Rotation Computations. J. Phys. Chem. A 2011, 115, 10045-10051.

(8) Srebro, M.; Govind, N.; de Jong, W. A.; Autschbach, J. Optical Rotation Calculated with Time-Dependent Density Functional Theory: The OR45 Benchmark. J. Phys. Chem. A 2011, 115, 10930-10949.

(9) Baranowska-Łączkowska, A.; Łączkowski, K. Z. The ORP Basis Set Designed for Optical Rotation Calculations. J. Comput. Chem. 2013, 34, 2006-2013.

(10) Schafer, A.; Horn, H.; Ahlrichs, R. Fully Optimized Contracted Gaussian Basis Sets for Atoms Li to Kr. J. Chem. Phys. 1992, 97, 25712577.

(11) Baranowska-Łączkowska, A.; Łączkowski, K. Z.; Henriksen, C.; Fernández, B.; Kozak, M.; Zielińska, S. New Basis Set for the Prediction of the Specific Rotation in Flexible Biological Molecules. RSC Adv. 2016, 6, 19897-19902.

(12) Coriani, S.; Baranowska, A.; Ferrighi, L.; Forzato, C.; Marchesan, D.; Nitti, P.; Pitacco, G.; Rizzo, A.; Ruud, K. Solvent 
Effects on the Conformational Distribution and Optical Rotation of gamma-Methyl Paraconic Acids and Esters. Chirality 2006, 18, 357369.

(13) Stephens, P. J.; Devlin, F. J.; Cheeseman, J. R.; Frisch, M. J. Calculation of Optical Rotation Using Density Functional Theory. J. Phys. Chem. A 2001, 105, 5356-5371.

(14) Grimme, S. Calculation of Frequency Dependent Optical Rotation Using Density Functional Response Theory. Chem. Phys. Lett. 2001, 339, 380-388.

(15) Ruud, K.; Helgaker, T. Optical Rotation Studied by DensityFunctional and Coupled-Cluster Methods. Chem. Phys. Lett. 2002, 352, 533-539.

(16) Autschbach, J.; Patchkovskii, S.; Ziegler, T.; van Gisbergen, S. J. A.; Baerends, E. J. Chiroptical Properties from Time-Dependent Density Functional Theory. II. Optical Rotations of Small to Medium Sized Organic Molecules. J. Chem. Phys. 2002, 117, 581-592.

(17) Egidi, F.; Carnimeo, I.; Cappelli, C. Optical Rotatory Dispersion of Methyloxirane in Aqueous Solution: Assessing the Performance of Density Functional Theory in Combination with a Fully Polarizable QM/MM/PCM Approach. Opt. Mater. Express 2015, 5, 196-209.

(18) London, F. Quantum Theory of Interatomic Currents in Aromatic Compounds. J. Phys. Radium 1937, 8, 397-409.

(19) Helgaker, T.; Ruud, K.; Bak, K. L.; Jørgensen; Olsen, J. Vibrational Raman Optical Activity Calculations Using London Atomic Orbitals. Faraday Discuss. 1994, 99, 165-180.

(20) Olsen, J.; Bak, K. L.; Ruud, K.; Helgaker, T.; Jørgensen. Orbital Connections for Perturbation-Dependent Basis Sets. Theor. Chim. Acta 1995, 90, 421-439.

(21) Miertus, S.; Scrocco, E.; Tomasi, J. Electrostatic Interaction of a Solute with a Continuum. A Direct Utilization of Ab Initio Molecular Potentials for the Prevision of Solvent Effects. Chem. Phys. 1981, 55, $117-129$.

(22) Cancés, E.; Mennucci, B.; Tomasi, J. A New Integral Equation Formalism for the Polarizable Continuum Model: Theoretical Background and Applications to Isotropic and Anisotropic Dielectrics. J. Chem. Phys. 1997, 107, 3032-3041.

(23) Mennucci, B.; Cancés, E.; Tomasi, J. Evaluation of Solvent Effects in Isotropic and Anisotropic Dielectrics and in Ionic Solutions with a Unified Integral Equation Method: Theoretical Bases, Computational Implementation, and Numerical Applications. J. Phys. Chem. B 1997, 101, 10506-10517.

(24) Frisch, M. J.; Trucks, G. W.; Schlegel, H. B.; Scuseria, G. E.; Robb, M. A.; Cheeseman, J. R.; Scalmani, G.; Barone, V.; Mennucci, B.; Petersson, G. A.; et al. Gaussian 09, Revision C.01; Gaussian Inc.: Wallingford, CT, 2010. 